\title{
A comparative molecular characterization of AMDV strains isolated from cases of clinical and subclinical infection
}

\author{
Marek Kowalczyk $^{1} \cdot$ Andrzej Jakubczak $^{1}$ (D) Beata Horecka ${ }^{1} \cdot$ Krzysztof Kostro $^{2}$
}

Received: 22 March 2018 / Accepted: 23 May 2018 / Published online: 29 May 2018

(c) The Author(s) 2018

\begin{abstract}
The Aleutian mink disease virus (AMDV) is one of the most serious threats to modern mink breeding. The disease can have various courses, from progressive to subclinical infections. The objective of the study was to provide a comparative molecular characterization of isolates of AMDV from farms with a clinical and subclinical course of the disease. The qPCR analysis showed a difference of two orders of magnitude between the number of copies of the viral DNA on the farm with the clinical course of the disease $\left(10^{5}\right)$ and the farm with the subclinical course $\left(10^{3}\right)$. The sequencing results confirm a high level of homogeneity within each farm and variation between them. The phylogenetic analysis indicates that the variants belonging to different farms are closely related and occupy different branches of the same clade. The in silico analysis of the effect of differences in the sequence encoding the VP2 protein between the farms revealed no effect of the polymorphism on its functionality. The close phylogenetic relationship between the isolates from the two farms, the synonymous nature of most of the polymorphisms and the potentially minor effect on the functionality of the protein indicate that the differences in the clinical picture may be due not only to polymorphisms in the nucleotide and amino acid sequences, but also to the stage of infection on the farm and the degree of stabilization of the pathogen-host relationship.
\end{abstract}

Keywords Aleutian disease $\cdot$ Molecular characterization $\cdot$ Phylogenetic analysis $\cdot$ Pathogen adaptation

\section{Introduction}

The main etiological agent of Aleutian mink disease is AMDV (Aleutian Mink Disease Virus), belonging to the family Parvoviridae and the genus Amdoparvovirus [1,2]. The target cells for replication of the virus are mainly $\mathrm{T}$ and B lymphocytes, and to a lesser extent macrophages. Replication of the virus stimulates a strong immune response in the body, causing pathological changes in the organs which lead to clinical disease symptoms, thereby reducing the profitability of mink farming due to small average litter size [3].

\section{Edited by Zhen F. Fu.}

Andrzej Jakubczak

andrzej.jakubczak@up.lublin.pl

1 Department of Biological Basis of Animal Production, Faculty of Biology, Animal Sciences and Bioeconomy, University of Life Sciences in Lublin, Akademicka 13, 20-950 Lublin, Poland

2 Department of Epizootiology and Clinic of Infectious Diseases, Faculty of Veterinary Medicine, University of Life Sciences, Głęboka 30, 20-612 Lublin, Poland
Elimination of mink infected with AMDV is as yet the only effective method of combating Aleutian disease on infected farms. It should be noted, however, that the virus survives for a long time in the living environment of infected mink [4], and therefore the introduction of animals free of AMDV infection to such a farm may result in further endemic persistence of the disease.

Routine diagnosis of Aleutian mink disease is primarily performed using serological methods based on immunoenzymatic assays (ELISA), immunoelectrophoresis (counterimmunoelectrophoresis-CIEP) or immunochromatographic assays [5]. Serological techniques, due to the high degree of antigenic and genetic variability of AMDV, are not always adequate [6] because they produce a certain percentage of false negative [7]. For this reason, molecular biology techniques such as PCR and its variants, isothermal amplification methods, and techniques based on pyrosequencing are increasingly used to identify AMDV [8]. The sensitivity and specificity of PCR techniques is higher than that of immunochromatographic analysis [9].

The course of Aleutian disease is determined by the age of the animal, its genotype and the virulence of the strain 
of virus. On Polish farms, the disease most often has a subclinical course [10], although cases with distinct clinical symptoms are noted as well. Therefore, the aim of the study was to provide a comparative molecular characterization of AMDV from a farm on which the course of Aleutian disease was clinical (farm A) and one with a subclinical course of the disease (farm B).

\section{Materials and methods}

\section{Experimental animals}

The study was carried out on two mink farms: the first located in north-western Poland (Farm A) and the second in north-eastern Poland (Farm B). The sanitary condition of the farm was assessed as satisfactory. The mink were fed in a traditional system according to recommendations for the species in the annual breeding cycle, including vitamin and mineral supplements, and they had continuous access to water. On farm A, with 10,000 female mink in the foundation herd, persistent AMDV infection with a clinical course was noted in $40 \%$ of the mink in the foundation herd, which was confirmed by counter-immunoelectrophoresis. Numerous deaths occurred on this farm, among symptoms including loss of appetite, weight loss, severe dehydration and bleeding from the mouth and anus, leading to severe anaemia, which was clinically manifested in pale mucous membranes and foot pads. Moreover, a fairly high percentage of unmated females and aborting females was observed, as well as high neonate mortality and a low average number of weaned kits. On farm B, with 5000 females in the foundation herd, persistent AMDV infection with a subclinical course was noted in $60 \%$ of the animals, also confirmed by counter-immunoelectrophoresis. In addition, a fairly high percentage of unmated and aborting females was noted, as well as high neonate mortality and a low average number of weaned kits.
The study was conducted on 20 female mink of the brown variety at the age of 1 year. Ten mink from each farm were used (groups A and B). Only females in which antibodies against AMDV were detected during specific diagnosis by counter-immunoelectrophoresis were selected for the study. Approval for these tests was obtained from the 2nd Local Ethics Committee for Experiments on Animals at the University of Life Sciences in Lublin (approval no. 83/2009).

\section{Serological diagnosis}

The biological material for serological diagnosis of AMDV consisted of blood samples collected into capillary tubes from living experimental animals by toenail clipping. The samples were tested by counter-immunoelectrophoresis, which is routinely used in diagnosis of Aleutian mink disease.

\section{Molecular diagnosis of AMDV}

The biological material for genetic testing consisted of blood samples collected by toenail clipping into tubes containing $5 \mathrm{mM}$ of EDTA as an anticoagulant. The genomic DNA of the virus was isolated using a kit for isolating DNA from blood (QIAamp DNA Investigator Kit, QIAGEN, Hilden, Germany), according to the manufacturer's instructions. The isolated DNA was used as a template for amplification by PCR (Labcycler thermal cycler, SensoQuest, Germany), which was conducted in triplicate, for three different primer pairs, i.e. two amplifying a fragment of the VP2 protein (primer pairs RP2 and RP3) [11] and one amplifying a fragment of the NS protein (primer pair NS) [12]. The sensitivity of the reaction was verified by qPCR. Table 1 presents the primer sequences and Table 2 shows the composition of the reaction mix and the temperature and time profile.

Qualitative assessment of AMDV was conducted by qPCR, using the Genesig Advanced Real-Time PCR Detection Kit for Aleutian Disease Virus, (PrimerDesign ${ }^{\mathrm{TM}} \mathrm{Ltd}_{\text {., }}$ UK) in an ABI Prism® 7500 Fast apparatus (Applied
Table 1 Primers and their annealing temperature

\begin{tabular}{|c|c|c|c|c|}
\hline Name of primer & Primer sequence 5'-3' & $\begin{array}{l}\text { Product } \\
\text { length (bp) }\end{array}$ & $\begin{array}{l}\text { Annealing tem- } \\
\text { perature }\left({ }^{\circ} \mathrm{C}\right)\end{array}$ & Refs. \\
\hline \multicolumn{5}{|l|}{ RP2 } \\
\hline Forward & TCTAGAAGCAACGCTTGGGGTGTATG & 802 & 58 & {$[11]$} \\
\hline Reverse & GTTGTGTCACTCCACTGTCT & & & \\
\hline \multicolumn{5}{|l|}{ RP3 } \\
\hline Forward & TCTAGATTGGGCCTACCТCСТCTCTG & 681 & 58 & \\
\hline Reverse & ATACAGGACCAACGTTGTCT & & & \\
\hline \multicolumn{5}{|l|}{ NS } \\
\hline Forward & KTTGGTTGCTTTACTCC & 500 & 54 & {$[12]$} \\
\hline Reverse & RTCTACTTTTACATCACCAC & & & \\
\hline
\end{tabular}




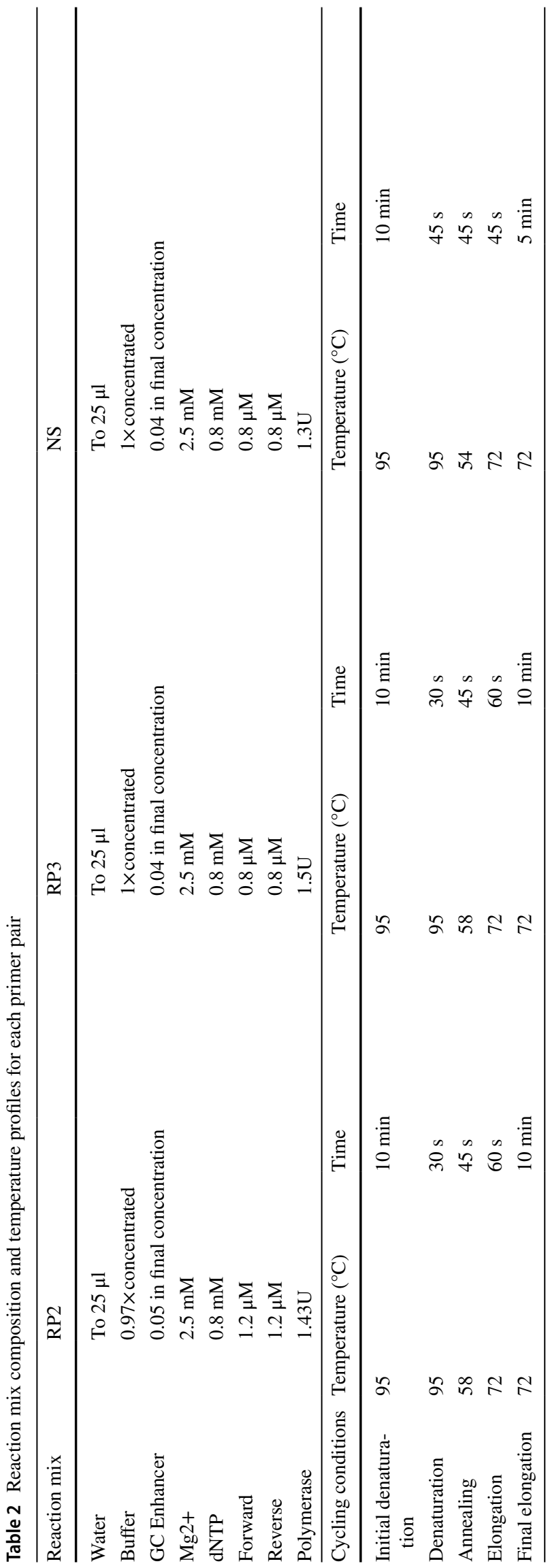

Biosystems, USA), according to the kit manufacturer's instructions. Serial dilutions of $2 \times 10^{5}, 2 \times 10^{4}, 2 \times 10^{3}$, $2 \times 10^{2}, 2 \times 10^{1}$ and $2 \times 10^{0}$ copies $/ \mu l$ were used to prepare the standard curve. These dilutions were prepared using the positive control supplied with the kit. The amount of product obtained for the samples was determined by comparing $\mathrm{Ct}$ values over the standard curve. The amount of DNA quantified for each sample was expressed as number of copies/ reaction. The standard curve was used to determine the number of copies of the virus based on the $\mathrm{Ct}$ value (as number of copies/reaction).

To confirm the PCR products, gel electrophoresis was carried out using $2 \%$ agarose gel with ethidium bromide $(\mathrm{EtBr})$. The PCR product was purified using an ExoSAP-IT kit (Affymetrix, California, USA). The second amplification (sequencing PCR)—bidirectional sequencing—was carried out with a BigDye ${ }^{\circledR}$ Terminator v3.1 Cycle Sequencing Kit (Applied Biosystems, USA), using the primers RP2 and RP3. PCR products were purified using anExTerminator kit (A\&A Biotechnology, Poland). PCR products were sequenced using a 3100 Genetic Analyzer (Applied Biosystems, USA). To avoid sequencing errors, both sequenced strands were analysed. In the case of doubtful samples, additional runs were prepared and comparisons were made with deposited sequences. The sequences were assembled into consensus sequences using DNA Baser [13].

Similarity between sequences was determined using BioEdit software, and localization of polymorphisms was determined with Mega 7 software. The phylogenetic distances were estimated with the Hasegawa, Kishino and Yano model (HKY), using the Model option in MEGA 7 software [14]. Phylogenetic relationships were reconstructed using a Bayesian algorithm in Mr Bayes software [15, 16]. Bayesian analysis with MrBayes used four linked Markov chains per run and two simultaneous runs. The convergence and stationarity of the runs were assessed by checking for plateaus in the time series of parameter values and examining the standard deviation of split frequencies between the two runs. The simulations were carried out for $5 \times 10^{5}$ generations, which were logged every 100 generations. The phylogram was presented in FigTree software (http://tree.bio.ed.ac.uk/ software/figtree/). A complementary sequence of the raccoon dog virus (KJ396347) was used as the outgroup.

\section{Results}

Sequences encoding the VP2 protein fragment were obtained for all mink in the experimental groups from farms A and B. For individuals for which amplification was obtained for primers RP2 and RP3, contigs were assembled to obtain a fragment with a combined length of $1138 \mathrm{bp}$. Amplification of the VP2 protein fragment 
using the RP2 primer pair revealed the presence of genetic material in all samples from farm A except for mink no. 4 (Fig. 1). The amplified sequence contained a hypervariable region, which may be the cause of false-negative results. The analysis conducted using the RP3 pairs flanking the sequence encoding the VP2 protein fragment produced a negative result for samples 2 and 4. Amplification using the NS primer pairs confirmed the presence of AMDV genetic material in all experimental mink from farm A (Fig. 1).

On farm B, genetic material of AMDV was detected by PCR in eight of the ten samples using the RP3 primer pair, while the presence of the viral DNA was confirmed in three samples for primer pair RP2 and in five samples in the case of the NS primers.

All samples with amplified genetic material of AMDV were subjected to qPCR. In the case of farm A, the amount of genetic material of the virus in the samples was comparable to the positive control supplied in the commercial kit for detection of AMDV (Fig. 2). The negative PCR results were verified by qPCR, which confirmed the positive result for mink no. 4 for the fragment encoding the NS protein, but the number of copies of viral DNA was significantly lower.
The results of amplification of the viral DNA in the farm $\mathrm{B}$ animals by qPCR confirmed the presence of AMDV for samples 1 and 2 (positive reaction for one primer pair in classical PCR) and a negative result was obtained in mink 9 and 10 (negative result for classical PCR) (Fig. 1).

Quantitative determination of the number of copies of the virus by qPCR in the case of the mink from farm B was at a level of $10^{3}$. The highest viral load was noticed in case of sample $8-1.69 \times 10^{4}$, the lowest number of copies on this farm was detected in sample $1-2.32 \times 10^{2}$. Quantitative determination of viremia in animals from farm $\mathrm{A}$ indicated that the mean number of copies was higher by two orders of magnitude, at $10^{5}$ (Fig. 2) with the highest number of copies in sample $10-7.11 \times 10^{5}$, and the lowest in sample $4-2.33 \times 10^{3}$ (Fig. 3). Statistical analysis confirmed that viral copy number was significantly higher on farm A in comparison to farm B.

The PCR products obtained for the mink with the clinical and subclinical course of Aleutian disease were sequenced. The sequences obtained for both primer pairs flanking the sequence encoding the VP2 protein were highly homogeneous within the farms, but differences were noted between farm A and farm B. SNP polymorphism was noted for
Farm A

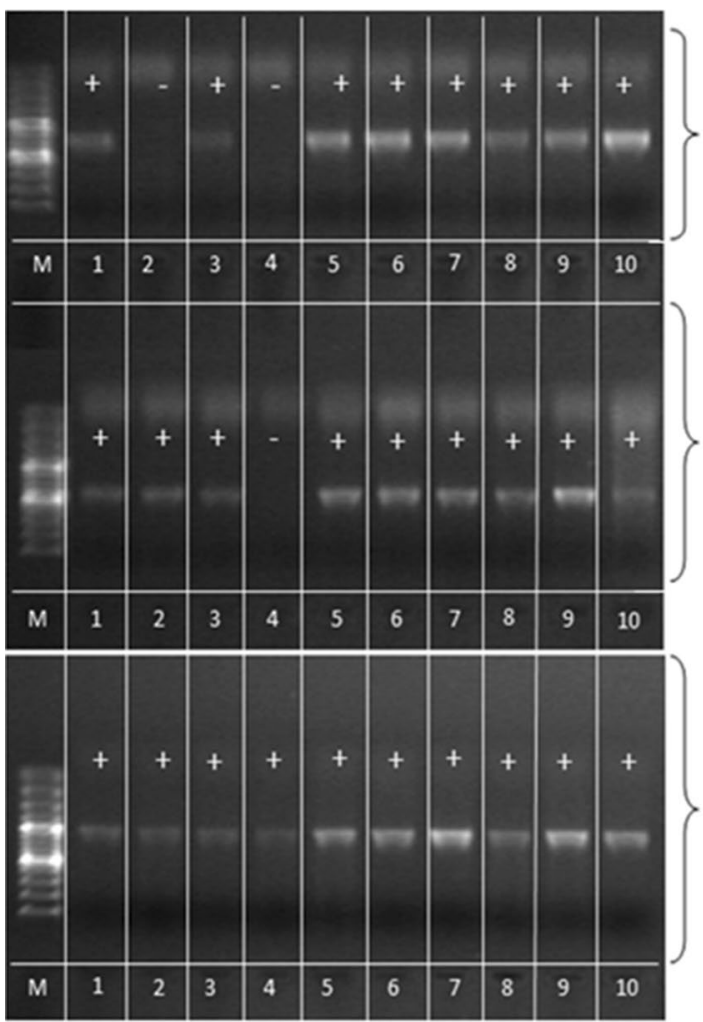

Farm B

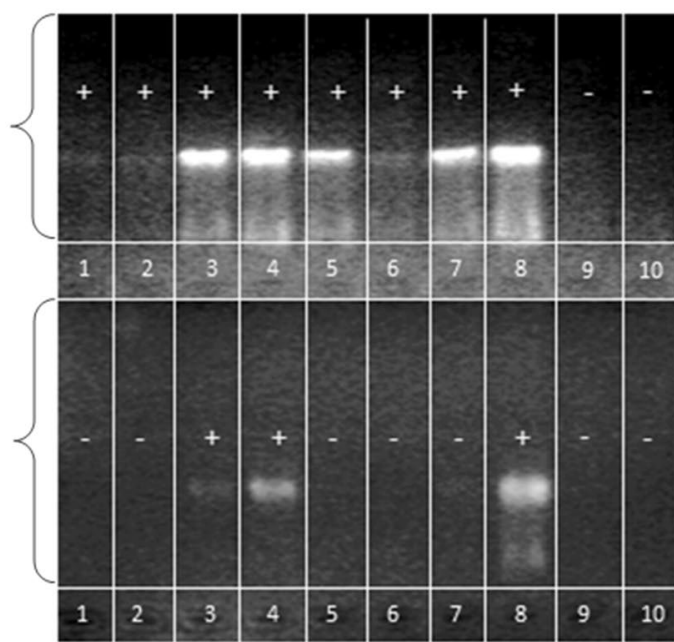

NS

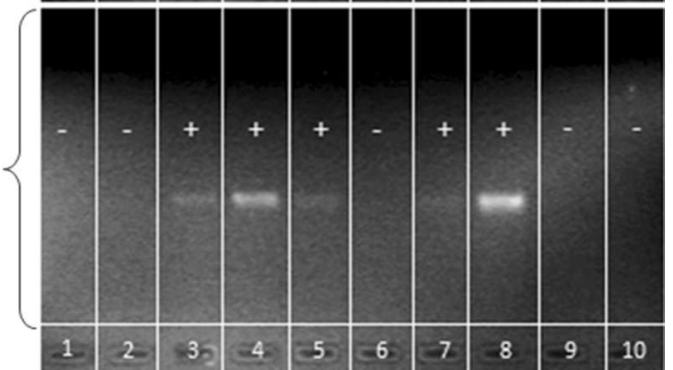

Fig. 1 Results of electrophoresis of PCR products for three primer pairs, RP2, RP3 and NS. Lane M-100 bp size marker, Farm A-lanes 1-10, Farm B-lanes 1-10 
Fig. 2 Result of qPCR analysis for farm A mink-virus titre similar to that of the positive control (order of magnitude $10^{5}$ )
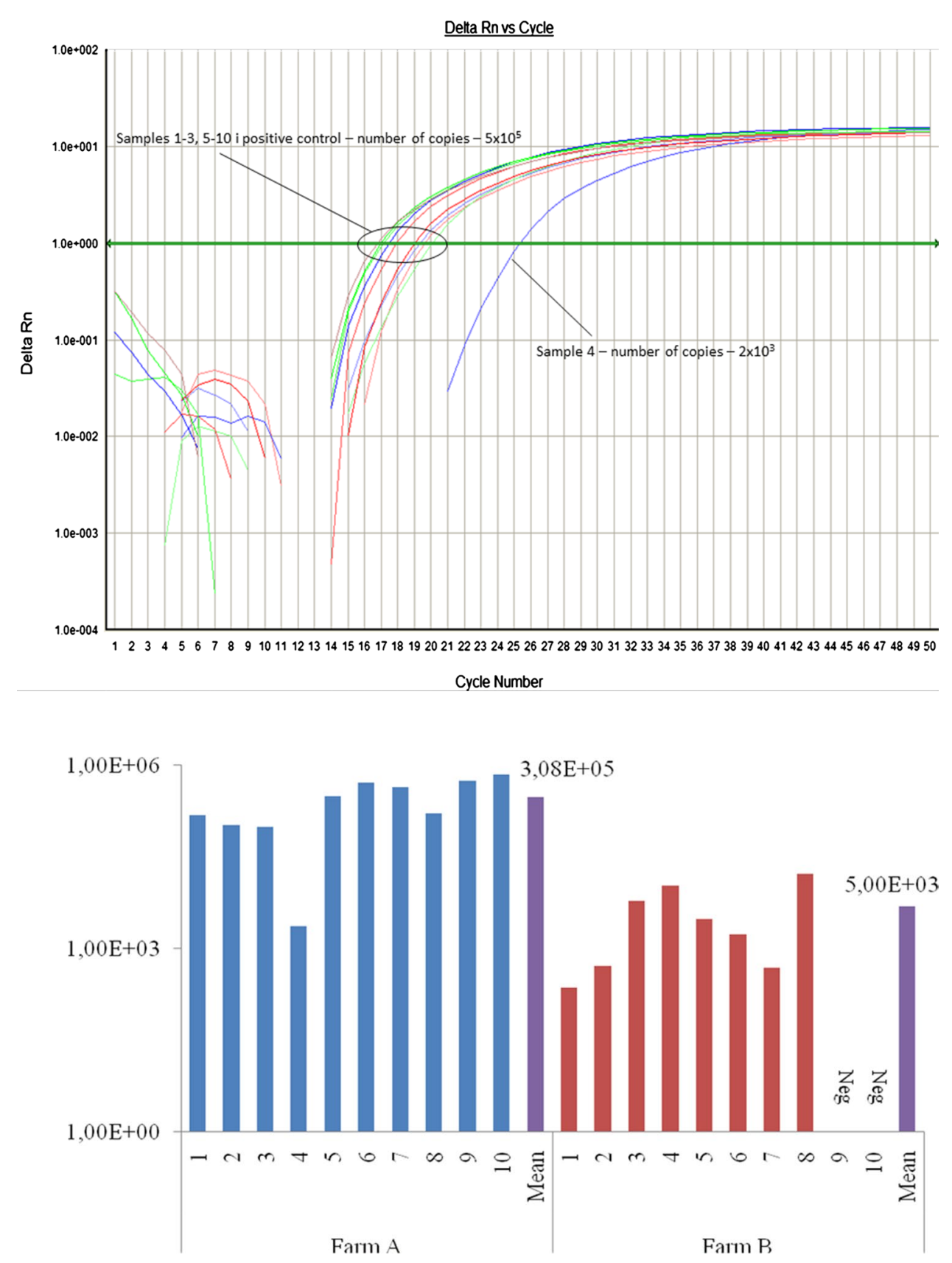

Fig. 3 Comparison of number of copies of AMDV genetic material in blood of animal from farm $\mathrm{A}$ - blue, and $\mathrm{B}$ red. (Color figure online) sample no. 2 from farm A, which indicates the occurrence of at least two variants on the farm, with the second variant being the effect of a single mutation in the genetic material of the virus initially infecting the farm. The PCR product was sequenced with the RP2 primer pair (negative result with primer pair RP3) and it was found to contain a mutation in position C3047T (Fig. 4). The lack of amplification product for the RP3 primer pair may have been due to polymorphisms at the primer binding site, which may explain the false-negative result.

Sequencing of isolates from the experimental mink from the two farms in the case of which amplification of the fragment encoding the VP2 protein was obtained for primer pairs RP2 and RP3 made it possible to identify differences in the genetic material of the virus between the two farms. There were 47 differences shown in the nucleotide sequence, including four in the hypervariable region, which was $4 \%$ of the entire analysed sequence. This corresponded to changes in 12 amino acids, with one change in the hypervariable region. The contigs obtained on both farms were compared with the apathogenic control strain AMDV-G. The occurrence of 78 differences was noted in the nucleotide sequence in the case of farm $\mathrm{A}$ and 87 for farm $\mathrm{B}$ in relation to the non-pathogenic strain, with much higher variability shown than in the case 
Fig. 4 a chromatogram showing the polymorphic nucleotide, b variants of fragments of the VP2 protein nucleotide sequence, $\mathbf{c}$ synonymous nature of the mutation

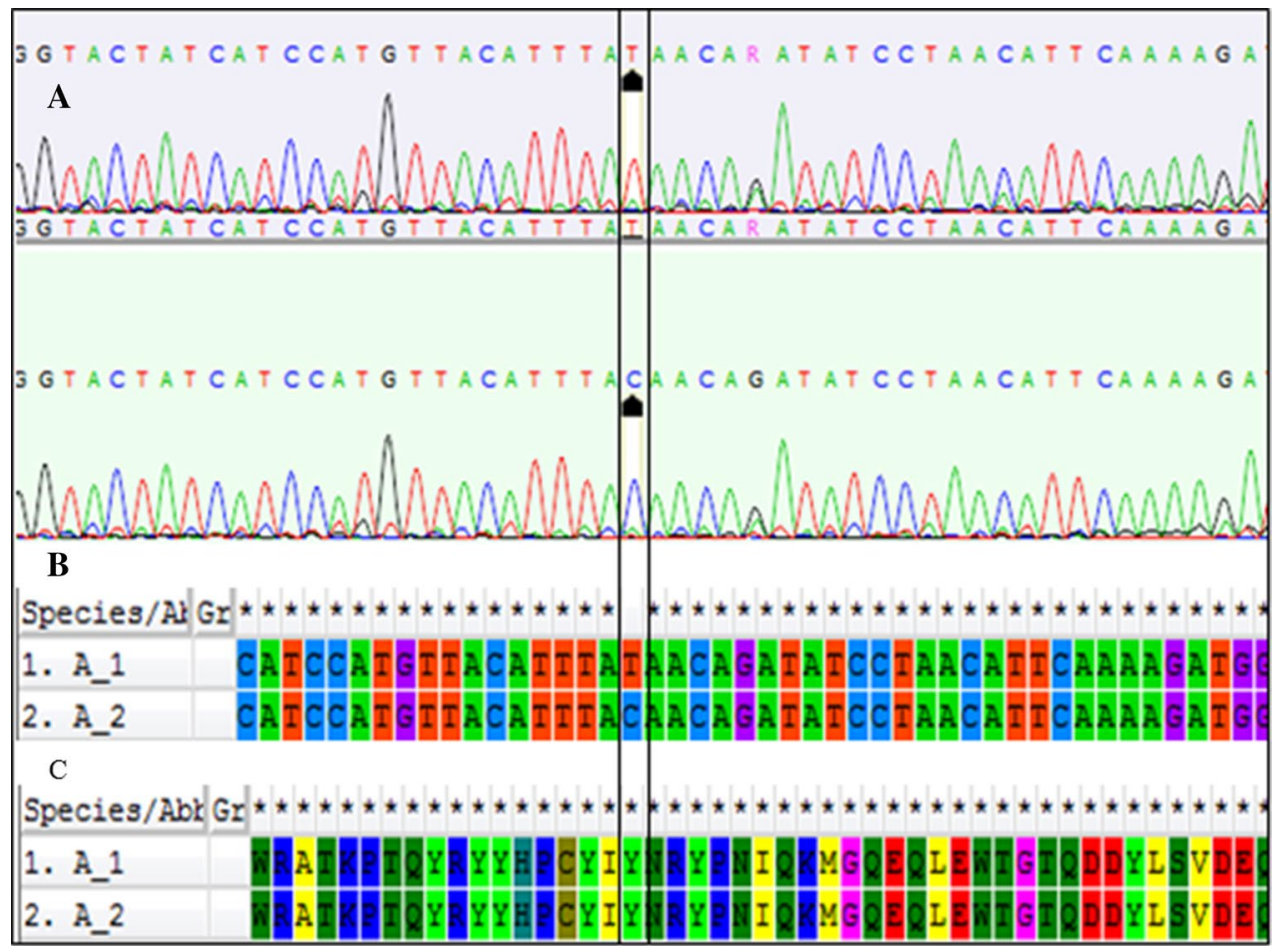

of the highly pathogenic Utah strains (44 differences), LN-1, LN-2 and LN-3 (40-50 differences) and lower variability than the for Pullman strain (91 differences).

The sequences obtained were compared with the main strains available in databases, i.e. Utah, AMDV-G, TR, LN-1, LN-2, LN-3 and Pullman, and with sequences of AMDV from other parts of the world. Their phylogenetic analysis made it possible to form three main groups (Fig. 5). All isolates obtained from farms A and B were assigned to the first group together with the Pullman reference strain. The second group comprised the highly pathogenic LN and Utah strains, while the third group consisted of the non-pathogenic strain AMDV$\mathrm{G}$, Russian strains and the highly pathogenic TR strain.

In silico analysis of the effect of polymorphisms in the amino acid sequence on the functionality of the VP2 protein indicates wide variation for AMDV without the risk of loss of functionality. Among the 31 differences in the amino acid sequence of the VP2 protein between isolates from farms A and $B$ and the apathogenic reference strain AMDV-G, only one change, Y350M, shows a potential effect on the functionality of the protein (Fig. 6). In the hypervariable region, eight differences were noted in the amino acid sequence, but none of them affected the functionality of the protein.

\section{Discussion}

Molecular methods have become extremely useful not only in diagnostics but also for understanding the phenomena associated with mutations in microorganisms. The study compared AMDV isolates from mink farms with clinical and subclinical forms of Aleutian disease. None of the primer pairs used in the study was able to conclusively identify the infection, which is confirmed by results obtained by Landry et al. [17]. In the authors' opinion, the primers designed for conserved regions of the viral genome have limited diagnostic value due to its mutational changes. Thus primers must be verified and updated, as new variants of viral sequences appear in bioinformatic databases. Therefore, one means of reducing the number of false-positive results is to design PCR reactions for several fragments of the viral genome, which is reflected in the results of the present study. This seems to be particularly important when there is a high concentration of farmed mink on an infected farm, because this is conducive to spread of the infection and the emergence of mutated forms of the same strain of the virus, 
Fig. 5 Comparison of phylogenetic relationships between the isolates and selected sequences from bioinformatic databases. Phylogenetic relationships were reconstructed using a Bayesian algorithm with MrBayes software using four linked Markov chains per run and two simultaneous runs. A complementary sequence of the raccoon dog virus (KJ396347) was used as the outgroup

Fig. 6 Prediction of functional effects of mutations prepared in the SNAP2 application, showing polymorphic amino acids in relation to the AMDV-G strain. Filled black squares-no change or synonymous substitution; empty black squares—substitution on both farms; empty blue squares-Farm A, empty orange squares-Farm B. Each substitution was shown independently for each position of the protein in a heatmap representation. Dark red indicates a high score (score $>50$, strong signal for effect), white indicates weak signals $(-50<$ score $<50)$ and green a low score (score $<-50$, strong signal for neutral/no effect). Black designates the corresponding wild-type residues. (Color figure online)

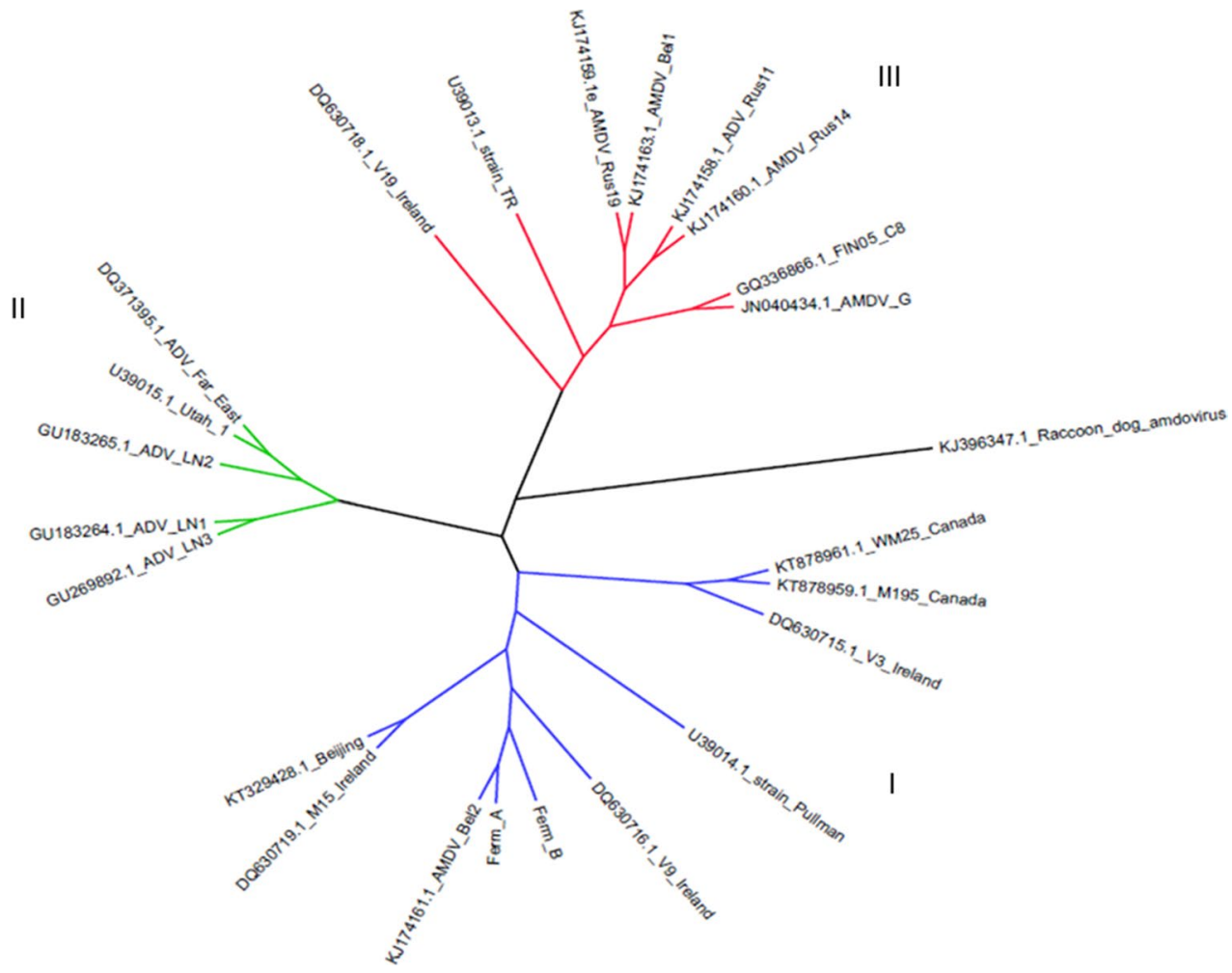

Hypervariable Region

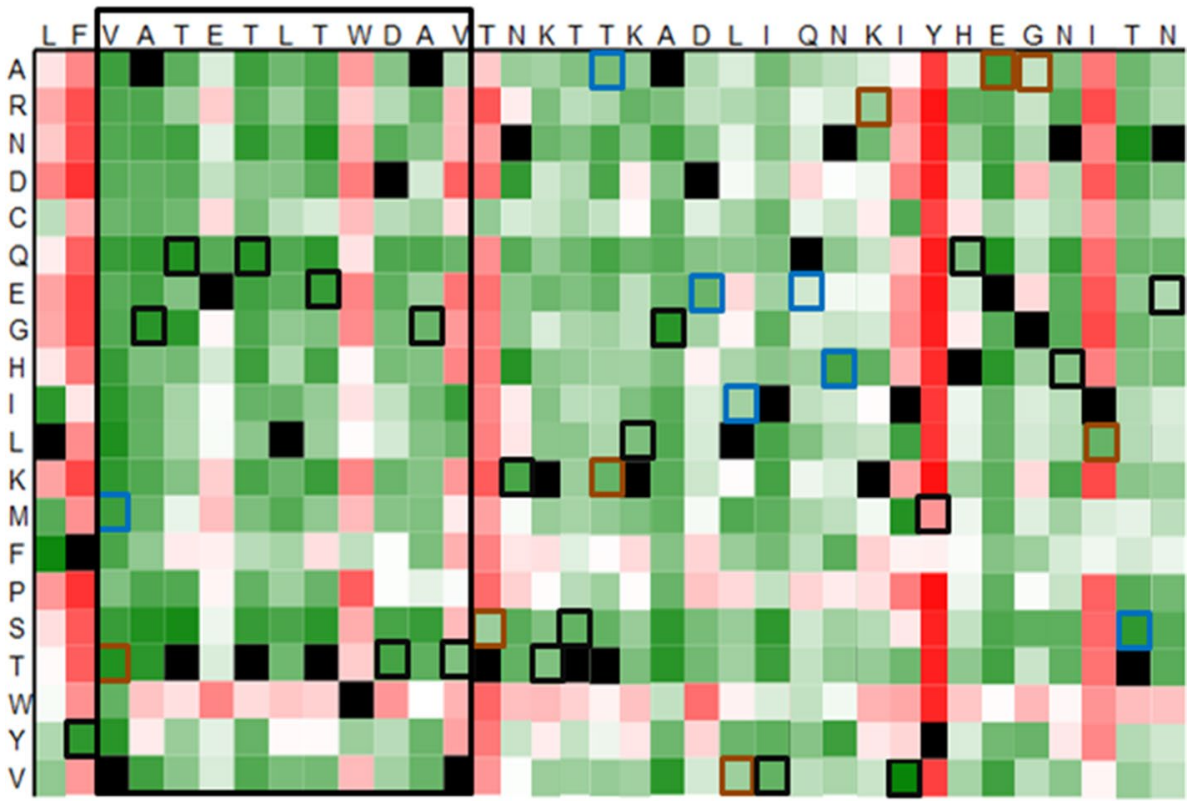

which can impede specific diagnosis of Aleutian disease by molecular biology methods. Thus the false-negative results obtained with the RP2 and RP3 primer pairs were probably not due to the sensitivity of the method, because the reaction yielded a positive result in the case of the NS primer pair. The lack of amplification may have been due to the occurrence of polymorphism at the primer binding site or a stage of infection in which the amount of material encoding the structural protein was below the method's detection threshold (Fig. 1).

The number of copies of the virus in the material is important both for the course of the disease itself and for 
the effectiveness of diagnostic measures. The dynamics of the pathogen-host relationship affect detection of the virus; a lower degree of penetration of the virus may be correlated with a lower titre of the pathogen in the tissues, making it more difficult to detect. A larger amount of the virus's genetic material indicates a faster replication rate, which may be both a cause and an effect of a specific form of the disease.

The in silico test of the effect of differences within the sequence encoding the VP2 protein between farms A and $B$ showed that polymorphism had no effect on its functionality. The difference noted in functionality with respect to the non-pathogenic strain (Y350M) may be due to different physical and chemical properties of tyrosine and methionine. A substitution by an amino acid with different physicochemical properties can affect the activity of the entire peptide. In the case of parvoviruses, polymorphism in the capsid protein sequence may significantly affect the pathogen's spectrum of infection [18]. The hypervariable region, having the most mutational changes, displays tolerance for changes in the amino acid sequence. High variability in the hypervariable region and its potential association with both pathogenicity in parvoviruses and protein functionality have been confirmed by Perez et al. [19], Saifuddin and Fox [20] and Majer-Dziedzic et al. [21]. The VP2 protein, containing a hypervariable region and epitopic regions, is largely responsible for the antigenic properties of the pathogen and its spectrum of infection [22, 23]. In the case of amdoviruses, which include AMDV, the variation between isolates is particularly high even within individual farms [24].

Large numbers of animals on farms, intensive breeding work, and a high mutation rate of the ssDNA of viruses [25] are conducive to the evolution of the pathogen and the generation of new variants [12], with varying degrees of adaptation to the occupied niche. In the case of farm A, where the disease had an aggressive course, the virus may be eliminated from the host population, due to both the high mortality rate of the infected animals and measures taken by breeders to eradicate it. On farm B, following colonization the pathogen became established in the population, inducing persistent, non-progressive infections.

Colonization of a new ecological niche by a pathogen often results in a rapid course, which is unfavourable not only for the host, but also for the pathogen. When an infectious agent encounters no immune resistance in the host, it rapidly replicates and spreads, inducing severe but shortlived outbreaks of the disease. Given the high stocking density of mink and thus the potential spread of the disease, the pathogen-host relationship should be considered not on an individual level, but at the level of the population. The aim of the virus is not to eliminate the host but to become established in the population and stabilize the pathogen-host relationship. The adaptive value of mutations in the structural protein VP2 of the canine parvovirus CPV-2 has been confirmed by Pereira et al. [26], who observed that the virus strives for maximum adaptation and establishment in the population.

Adaptive changes which may appear in new variants may be preferred by natural selection to the extent that the new form completely supplants the original strain. This occurred in the case of the canine parvovirus (CPV-2), where the original CPV-2 strain was completely replaced by the variant CPV-2a, which had the additional ability to persist in the organism of other dogs and in cats as well [19]; moreover, further mutations resulted in the emergence of variants CPV-2b and CPV-2c [21]. In the case of parvoviruses, minor changes in the amino acid sequence can diametrically alter the properties of the pathogen. This may have occurred in the case of CPV-2, whose ancestor was likely the feline panleukopenia virus (FPV) [27]. The VP2 protein, containing a hypervariable region and epitopic regions, is largely responsible for the antigenic properties of the pathogen and the range of species it infects [23]. In this way, variability within it can play a crucial role in adaptation of the virus to a given environment.

The development of pathogen-host equilibrium on AMDV-infected farms is confirmed by Farid and Ferns [28], who also showed that histopathological changes are smaller in the case of animals selected for resistance to AMDV. Results reported by Bloom et al. [29] also indicate the possibility of a gradual stabilization of the pathogen-host relationship. The authors showed that some colour varieties of mink display a certain tolerance for AMDV, and infection in these animals takes a persistent, non-progressive form. A non-persistent infection with gradual suppression of replication of the virus may occur as well.

The present study showed that the AMDV isolates from farm A are closely related, which is confirmed by the minor variation between them in the sequence of the hypervariable region, and fact that the single mutation is synonymous. Pereira et al. [26] observed that synonymous mutations in the VP structural protein, despite having no effect on capsid structure, may take part in epistatic interactions. On farm B, on the other hand, the same variant of the pathogen was diagnosed in all individuals, which given the subclinical course of the infection and the low mortality rate may indicate the establishment of a state of equilibrium between the host and the virus. The basic reproduction number $R_{0}$, representing the number of secondary infections from the original host, has an important role in maintenance of the pathogen in the host population. From an evolutionary point of view, genetic variants of the pathogen with higher values for $R_{0}$ are preferred [12]. In the early stages of infection, the population of the pathogen increases rapidly, but over time the rate of its spread stabilizes. The period of rapid spread is associated with the emergence of many different, often 
unique variants of the pathogen, which may be an attempt to adapt to the new niche [26].

\section{Conclusions}

To conclude, the molecular methods applied made it possible to characterize the genetic material of AMDV in isolates from two farms with different clinical courses of Aleutian disease. The results confirm high variability in the virus, which necessitates continual updating and verification of primers. Furthermore, it also appears essential to amplify several conserved fragments of the viral genome at the same time. Molecular analysis of the isolates revealed a high degree of homogeneity within the farms, but it may have various causes. Given the close phylogenetic similarity of the two variants, the synonymous nature of most of the polymorphisms and their potentially minor effect on the functionality of the protein, the differences in the clinical picture may be due not only to differences in the nucleotide and amino acid sequences, but also to the stage of infection on the farm and the degree of stabilization of the pathogen-host relationship.

Author contributions $\mathrm{MK}$ and $\mathrm{AJ}$ conceived and planned the experiments. MK and BH contributed to laboratory and bioinformatic analysis. MK and AJ drafted the manuscript. AJ and KK supervised the research. Manuscript was approved by all the authors.

\section{Compliance with ethical standards}

Conflict of interest The authors declare that there is no conflict of interest.

Ethics approval Approval for these tests was obtained from the 2nd Local Ethics Committee for Experiments on Animals at the University of Life Sciences in Lublin (Approval No. 83/2009).

Open Access This article is distributed under the terms of the Creative Commons Attribution 4.0 International License (http://creativeco mmons.org/licenses/by/4.0/), which permits unrestricted use, distribution, and reproduction in any medium, provided you give appropriate credit to the original author(s) and the source, provide a link to the Creative Commons license, and indicate if changes were made.

\section{References}

1. E.E. Hagberg, A. Krarup, U. Fahnoe, L.E. Larsen, R. Dam-Tuxen, A.G. Pedersen, J. Virol Methods 234, 43-51 (2016)
2. A. Leimann, A. Knuuttila, T. Maran, O. Vapalahti, U. Saarma, Virus Res. 199, 56-61 (2015)

3. T.H. Jensen, M. Chriel, M.S. Hansen, Acta Vet. Scand. 58(1), 35 (2016)

4. A. Kramer, I. Schwebke, G. Kampf, BMC Infect Dis. 6, 130 (2006)

5. D. Taylor, M. Durigon, H. Davis, C. Archibald, B. Konrad, D. Coombs, M. Gilbert, D. Cook, M. Krajden, T. Wong, G. Ogilvie, Int J STD\& AIDS 26(4), 215-224 (2015)

6. L. Yan, L.W. Pace, B. Baughman, F.D. Wilson, S. Zhang, M.Z. Zhang, J. Vet. Diagn. Invest. 28(2), 150-157 (2016)

7. Z. Chen, Y. Wang, Z. Wang, Y. Ke, Q. Zhen, X. Yuan, W. Zhang, Y. Lu, Y. Yu, H. Song, L. Huang, Clin. Infect. Dis. 57(2), 322-323 (2013)

8. P.N. Bernardino, S.M. Mapes, R. Corbin, N. Pusterla, J. Vet. Diagn. Invest. 28(3), 323-326 (2016)

9. T.M. Uyeki, R. Prasad, C. Vukotich, S. Stebbins, C.R. Rinaldo, Y. Ferng, S.S. Morse, E.L. Larson, A.E. Aiello, B. Davis, A.S. Monto, Clin. Infect. Dis. 48(9), 89-92 (2009)

10. M. Reichert, K. Kostro, Bull. Vet. Inst. Pulawy 58(3), 369-373 (2014)

11. F. Costello, N. Steenfos, K.T. Jensen, J. Christensen, E. Gottschalck, A. Holm, B. Aasted, Scand. J. Immunol. 49(4), 347-354 (1999)

12. M. Canuti, K.E. O'Leary, B.D. Hunter, G. Spearman, D. Ojkic, H.G. Whitney, A.S. Lang, Virus Evol. 2(1), 1-17 (2016)

13. HeracleBioSoft. http://www.DnaBaser.com

14. S. Kumar, G. Stecher, K. Tamura, Mol. Biol. Evol. 33(7), 18701874 (2016)

15. J.P. Huelsenbeck, F. Ronquist, Bioinformatics 17(8), 754-755 (2001)

16. F. Ronquist, J.P. Huelsenbeck, Bioinformatics 19(12), 1572-1574 (2003)

17. M.L. Landry, T. Eid, S. Bannykh, E. Major, J. Clin. Virol. 43(2), 247-249 (2008)

18. R.M. Soares, A. Cortez, M.B. Heinemann, S.M. Sakamoto, V.G. Martins, M. Bacci, F.M. De Campos Fernandes, L.J. Richtzenhain, J. Gen. Virol. 84, 1505-1515 (2003)

19. R. Perez, P. Bianchi, L. Calleros, L. Francia, M. Hernandez, L. Maya, Y. Panzera, K. Sosa, S. Zoller, Vet. Microbiol. 155(2-4), 214-219 (2012)

20. M. Saifuddin, J.G. Fox, Arch Virol. 141(7), 1329-1336 (1996)

21. B. Majer-Dziedzic, A. Jakubczak, J. Zietek, Pol. J. Vet. Sci. 14(3), 379-384 (2011)

22. J.M. Raj, H.K. Mukhopadhyay, J. Thanislass, P.X. Antony, R.M. Pillai, Infect. Genet. Evol. 10(8), 1237-1241 (2010)

23. L. Yi, Y.N. Cheng, M. Zhang, Z.G. Cao, M.W. Tong, S.P. Cheng, X. Yan, Virus Res. 223, 39-42 (2016)

24. A. Jakubczak, M. Kowalczyk, K. Kostro, B. Horecka, G. Jezewska-Witkowska, Acta Virol 60(4), 354-360 (2016)

25. L.A. Shackelton, C.R. Parrish, U. Truyen, E.C. Holmes, Proc. Natl. Acad. Sci. USA 102(2), 379-384 (2005)

26. C.A.D. Pereira, E.S. Leal, E.L. Durigon, Infect. Genet. Evol. 7(3), 399-409 (2007)

27. U. Truyen, Vet. Microbiol. 117(1), 9-13 (2006)

28. A.F. Farid, L.E. Ferns, Res. Vet. Sci. 111, 127-134 (2017)

29. M.E. Bloom, H. Kanno, S. Mori, J.B. Wolfinbarger, Infect. Agents Dis. 3(6), 279-301 (1994) 